\title{
Dolutegravir for pregnant women living with HIV
}

\author{
Jonathan Zipursky MD, Mona Loutfy MD MPH
}

- Cite as: CMAJ 2020 March 2;192:E217-8. doi: 10.1503/cmaj.191227

I

n 2018, major health agencies, including Health Canada, the World Health Organization (WHO), and the US Food \& Drug Administration, issued drug safety warnings recommending against prescribing the antiretroviral drug dolutegravir to pregnant women. Concerns stemmed from an interim analysis of a Botswanan surveillance study that showed a nearly sixfold relative increase in neural tube defects in children born to women taking dolutegravir compared with other antiretrovirals. ${ }^{1}$ However, final data from the study of 119033 newborns were reported a year later, revealing a smaller (threefold) relative risk difference and an absolute risk difference of $0.2 \%$ (95\% confidence interval 0.01-0.59). ${ }^{2}$ The updated results prompted the WHO to reverse its initial stance and recommend dolutegravir as a preferred first- and second-line treatment for all populations living with HIV, including pregnant women and those of reproductive age. However, Health Canada has yet to reconsider its recommendation, which creates a dilemma for providers and patients when choosing which combination of antiretroviral drugs to prescribe for pregnant women.

Dolutegravir is an integrase inhibitor that works by inhibiting the insertion of HIV DNA into host cells, thereby preventing ongoing viral replication. ${ }^{3}$ It is considered an important antiretroviral therapy for many patients living with HIV because it has a favourable adverse effect profile and few drug-drug interactions, is taken once daily as part of a multidrug regimen, and confers a low risk of developing resistance compared with other antiretrovirals. Moreover, in 2017, the fixed-dose combination pill including dolutegravir, lamivudine and tenofovir was made available in generic form, making for a convenient and costefficient approach to antiretroviral treatment (the annual cost of this single pill regimen is US\$75 per patient in low-income countries). ${ }^{4}$ In high-income countries such as Canada, dolutegravir is also appealing for many women living with HIV and their care providers - particularly women with issues such as food and/or housing insecurity and substance use - because of its ease of use and high barrier to resistance.

A biologically plausible mechanism linking dolutegravir to neural tube defects, which may help to explain the findings of the Botswanan study, has recently been uncovered. ${ }^{5}$ Recent research using in vitro and animal models suggested that

\section{KEY POINTS}

- A recent observational study from Botswana reported a small increased risk of neural tube defects in children born to women living with HIV who were taking dolutegravir compared with other antiretrovirals.

- Owing to the high resistance barrier, favourable adverse effect profile, convenient administration and low cost of dolutegravir, possible fetal risks should be balanced against the potential for improved maternal health and reduced HIV vertical transmission.

- Dolutegravir should be considered an appropriate treatment option for women living with HIV who are pregnant or considering pregnancy if they are carefully engaged and involved in decision making about their antiretroviral treatment.

- If a woman is pregnant, plans to become pregnant, or is not using effective contraception while taking dolutegravir, we advocate for prescribing higher doses of folic acid (e.g., $5 \mathrm{mg} / \mathrm{d}$ ).

dolutegravir exerts partial antagonism at a folate receptor important for neural tube development, but also showed that this antagonism can be overcome by administering higher doses of folate. ${ }^{5}$ Botswana does not mandate folate-fortified grains; in the index study less than $1 \%$ of women were prescribed folate before conception and only $49 \%-69 \%$ were prescribed folate at any time during pregnancy. ${ }^{1,2}$ Therefore, the increased risk of neural tube defects observed in the Botswanan study might reflect inadequate folate supplementation rather than just teratogenicity from dolutegravir. ${ }^{5}$ Differences in nutritional resources and access to medical care in Botswana compared with other parts of the world limits the generalizability of the findings of the study to other populations.

Moreover, the absolute risk difference of $0.2 \%$ shown in the final analysis of the Botswanan study data corresponds to a number needed to harm of 500 . It bears mentioning that a single additional neural tube defect detected in the group that was not exposed to dolutegravir would have voided statistical significance. Another study conducted in Botswana ${ }^{6}$ and a 2018 systematic review ${ }^{7}$ showed no association between dolutegravir and congenital anomalies. Although no other antiretrovirals are 
currently associated with neural tube defects, many have not been studied rigorously in pregnancy.

Accumulating evidence and changing guidance regarding dolutegravir echoes the early drug safety warnings about efavirenz. Based on preliminary data in 2010, treatment guidelines recommended nevirapine over efavirenz because of an association between the latter drug and neural tube defects. Years later, however, updated data revealed no such association. ${ }^{8}$

While neural tube defects can lead to substantial morbidity and mortality, keeping maternal HIV under excellent control during pregnancy is important both for maternal health and because the risk of vertical transmission without viral suppression is high - between $15 \%-30 \% .^{9}$ Shared decisions about dolutegravir use in pregnancy should be made with patients by balancing risks and benefits to both mother and baby, and considering the known association between the drug's use and neural tube defects in light of other risk factors for such pathology (e.g., folic acid deficiency, poorly controlled diabetes, and maternal body habitus that might affect the ability to detect defects). Providers should discuss with patients the potential benefits of dolutegravir, such as its high barrier to viral resistance, which could improve maternal health and reduce HIV vertical transmission. Given the nuances of risks and benefits, providers should actively engage patients in decisions about their HIV treatment, particularly in the decision to start, continue or switch specific drugs. This is supported by a recent statement on dolutegravir issued by the Society of Obstetricians and Gynaecologists of Canada, and reflects the 2017 WHO consolidated guideline on the sexual and reproductive health and rights of women living with HIV. ${ }^{10,11}$

In patients with whom a decision is made to start or switch drugs preconception, HIV should be under adequate control for at least 3 months with at least 2 viral load measurements below the level of detection (at least 1 month apart) before pregnancy is attempted. ${ }^{12}$ In addition, the importance of prescribing preconception folic acid to women living with HIV cannot be overstated. Current guidelines for folic acid supplementation are similar to those for pregnant women living without HIV. ${ }^{12}$ However, if a woman plans to become pregnant or is not using effective contraception while taking dolutegravir, we advocate for prescribing higher doses of folic acid (e.g., $5 \mathrm{mg} / \mathrm{d}$ ).

Drug safety decisions in pregnancy are often mired in uncertainty; prescribing dolutegravir is no exception. However, given updated evidence and revised guidance from the WHO, we believe that dolutegravir should be considered an appropriate option for many women living with HIV who are pregnant or considering pregnancy if they are carefully engaged and decision making about their antiretroviral treatment is shared.

\section{References}

1. Zash R, Makhema J, Shapiro RL. Neural-tube defects with dolutegravir treatment from the time of conception. N Engl J Med 2018;379:979-81.

2. Zash R, Holmes L, Diseko M, et al. Neural-tube defects and antiretroviral treatment regimens in Botswana. N Engl J Med 2019;381:827-40.

3. Kandel CE, Walmsley SL. Dolutegravir - a review of the pharmacology, efficacy, and safety in the treatment of HIV. Drug Des Devel Ther 2015;9:3547-55.

4. New high-quality antiretroviral therapy to be launched in South Africa, Kenya and over 90 low- and middle-income countries at reduced price [press release]. Geneva: UNAIDS; 2017. Available: www.unaids.org/en/resources/presscentre/ pressreleaseandstatementarchive/2017/september/20170921_TLD (accessed 2020 Jan. 13).

5. Cabrera RM, Souder JP, Steele JW, et al. The antagonism of folate receptor by dolutegravir: developmental toxicity reduction by supplemental folic acid. AIDS 2019;33:1967-76.

6. Zash R, Jacobson D, Mayondi G, et al. Dolutegravir/tenofovir/emtricitabine (DTG) TDF/FTC) started in pregnancy is as safe as efavirenz/tenofovir/emtricitabine (EFV/ TDF/FTC) in nationwide birth outcomes surveillance in Botswana [abstract MOAX0202LB]. Proceedings of the International AIDS Society (IAS) Conference on HIV Pathogenesis Treatment and Prevention; 2017 July 23-26; Paris, France.

7. Hill A, Clayden P, Thorne C, et al. Safety and pharmacokinetics of dolutegravir in HIV-positive pregnant women: a systematic review. J Virus Erad 2018;4:66-71.

8. Ford N, Mofenson L, Shubber Z, et al. Safety of efavirenz in the first trimester of pregnancy: an updated systematic review and meta-analysis. AIDS 2014;28(Suppl 2): S123-31.

9. Volmink J, Marais B. HIV: mother-to-child transmission. BMJ Clin Evid 2008; 2008:pii: 0909.

10. Consolidated guideline on sexual and reproductive health and rights of women living with HIV. Geneva: World Health Organization; 2017.

11. SOGC Infectious Diseases Committee statement on dolutegravir use in pregnancy. The Society of Obstetricians and Gynaecologists of Canada (SOGC); 2019. Available: www.sogc.org/en/content/featured-news/Copy_of_sogc -infectious-diseases-committee-statement-on-dolutegravir-use-in-pregnancy. aspx (accessed 2020 Jan. 13).

12. Loutfy M, Kennedy VL, Poliquin V, et al. No. 354 - Canadian HIV pregnancy planning guidelines. J Obstet Gynaecol Can 2018;40:94-114.

Competing interests: Mona Loutfy has been a site investigator and has received funding for investigator-driven studies from ViiV Healthcare, Merck Frosst and Gilead Sciences in the past 5 years. Jonathan Zipursky has no conflicts of interest to disclose.

This article has been peer reviewed.

Affiliations: Division of Clinical Pharmacology and Toxicology (Zipursky), Department of Medicine, and Institute of Health Policy, Management, and Evaluation (Zipursky), University of Toronto; Women's College Research Institute (Loutfy); Division of Infectious Diseases (Loutfy), Department of Medicine, University of Toronto, Toronto, Ont.

Contributors: Jonathan Zipursky and Mona Loutfy contributed equally to the conception and design of the work. Jonathan Zipursky drafted the manuscript. Both authors revised the manuscript critically for important intellectual content, gave final approval of the version to be published and agreed to be accountable for all aspects of the work.

Correspondence to: Jonathan Zipursky, jonathan.zipursky@mail. utoronto.ca 